Please do not remove this page

RMIT

UNIVERSITY

\title{
Dynamic rerouting of vehicles during cooperative wildfire response operations
}

Van Der Merwe, Schagne-Kenneth; Ozlen, Melih; Hearne, John; Minas, James

https://researchrepository.rmit.edu.au/esploro/outputs/9921860473801341/filesAndLinks?institution=61RMIT_INST\&index=null

Van Der Merwe, S.-K., Ozlen, M., Hearne, J., \& Minas, J. (2017). Dynamic rerouting of vehicles during cooperative wildfire response operations. Annals of Operations Research, 254(1-2), 467-480.

https://doi.org/10.1007/s10479-017-2473-8

Document Version: Accepted Manuscript

Published Version: https://doi.org/10.1007/s10479-017-2473-8

Repository homepage: https://researchrepository.rmit.edu.au

(C) 2017, Springer Science+Business Media New York.

Downloaded On 2023/04/26 20:29:20 +1000

Please do not remove this page 
Thank you for downloading this document from the RMIT Research Repository.

The RMIT Research Repository is an open access database showcasing the research outputs of RMIT University researchers.

RMIT Research Repository: http://researchbank.rmit.edu.aul

\section{Citation:}

Van der Merwe, M, Ozlen, M, Hearne, J and Minas, J 2017, 'Dynamic rerouting of vehicles during cooperative wildfire response operations', Annals of Operations Research, vol. 254, no. 12, pp. 467-480.

See this record in the RMIT Research Repository at:

https://researchbank.rmit.edu.au/view/rmit:44504

Version: Accepted Manuscript

\section{Copyright Statement:}

(c) 2017, Springer Science+Business Media New York.

Link to Published Version:

https://dx.doi.org/10.1007/s10479-017-2473-8 


\title{
Dynamic rerouting of vehicles during cooperative wildfire response operations
}

\author{
Martijn van der Merwe • Melih Ozlen • John W. Hearne • \\ James P. Minas
}

\begin{abstract}
Incident managers assigning wildfire response vehicles to provide protection to community assets may experience disruptions to their plans arising from factors such as changes in weather, vehicle breakdowns or road closures. We develop an approach to rerouting wildfire response vehicles once a disruption has occurred. The aim is to maximise the total value of assets protected while minimising changes to the original vehicle assignments. A number of functions to measure deviations from the original plans are proposed. The approach is demonstrated using a realistic fire scenario impacting South Hobart, Tasmania, Australia. Computational testing shows that realistic sized problems can be solved within a reasonable time using a commercial solver.
\end{abstract}

Keywords integer programming · vehicle routing · dynamic rerouting · wildfire

M. van der Merwe

School of Science, RMIT University, GPO Box 2476, Melbourne, Victoria, 3001, Australia

Tel.: +61399253767

E-mail: martijn.vandermerwe@rmit.edu.au

M. Ozlen

School of Science, RMIT University, GPO Box 2476, Melbourne, Victoria, 3001, Australia

J.W. Hearne

School of Science, RMIT University, GPO Box 2476, Melbourne, Victoria, 3001, Australia

J.P. Minas

School of Business, State University of New York at New Paltz, New Paltz NY, 12561, USA 


\section{Introduction}

Uncontrolled wildfires occurring on populated landscapes can cause loss of life and damage to private property and community assets. In many jurisdictions including Australia, Canada, and the United States, incident management teams (IMTs) are responsible for coordinating, planning, and managing wildfire response related activities (Australasian Fire Authorities Council, 2013; ICS Canada, 2012; US Department of Homeland Security, 2008). These IMTs assign personnel and equipment to activities such as fire suppression and asset protection, and must make complex, time-critical decisions under dynamic, changing conditions.

During the February 7th 2009 Black Saturday wildfires, a number of fires burned out of control in South East Australia and resulted in the loss of 173 lives and of several thousand homes. A major contributing factor to the devastation caused by these fires was a change in wind direction, from north-westerly to south-easterly, that occurred late in the day. This wind change had a dramatic effect on fire orientation and resultant impact. A point in case being the Kilmore East fire. This was initially a long narrow fire band, but following the wind change the flank of the fire become a $55 \mathrm{~km}$ wide fire-front that burned through a number of townships with tragic consequences (Cruz et al., 2012). We are concerned with the development of a modelling approach to aid in reallocation of emergency resources in response to such changes in conditions.

In this paper we present an approach to assist IMTs in assigning resources to community asset protection activities during escaped wildfires. The wildfire asset protection problem was first modelled by Van der Merwe et al. (2015). They developed a mixed integer programming model which generalised the team orienteering problem with time windows (TOPTW) by adding heterogeneous vehicles and cooperative service requirements. They did not consider changes, disruptions or a dynamic approach to deal with changing conditions. Here we extend the approach proposed by Van der Merwe et al. (2015) to address the problem of rerouting emergency vehicles to adapt to changing weather conditions and other disruptions such as vehicle breakdowns and changes to the road network due to the impact of fire.

The problem of replanning and rerouting vehicles to deal with disruptions falls in the class of dynamic vehicle routing problems. Considerable attention has been given to dynamic vehicle routing problems which is evident from the review by Pillac et al. (2013). However, comparatively fewer studies have considered the rerouting of orienteering problems. Minis et al. (2011) considered the rerouting of delivery vehicles when breakdowns occur, modelling the problem as a dynamic team orienteering problem with time windows. Mamasis et al. (2012) formulated a rerouting team orienteering problem and provided a heuristic solution method. Murray and Karwan (2010) developed a mixed integer programming model for rerouting unmanned aerial vehicles when "pop-up" events occur. Pop-up events are new tasks or changes which were not planned for in the initial routing of the vehicles. Murray and Karwan (2010) provided a modelling framework which can model a wide variety of vehicle routing problems, including the TOPTW. A follow up paper by the same authors presented a branch-and-bound based method for solving their unmanned aerial vehicle assignment model (Murray and Karwan, 2013).

The vehicle rerouting studies by Minis et al. (2011) and Mamasis et al. (2012) did not consider the cooperative delivery of service, which is often required in wildfire response services. Our approach is most similar to that of Murray and Karwan (2010). However, we do not consider an infinite resource decision variable for infeasible assignments. Murray and Karwan (2010) have multiple nodes, each representing a task to be performed at the location, associated with a single location. Whereas, we consider only a single node for each location, thus reducing the number of integer decision variables required to describe the model. Further, we consider continuous time as opposed to discrete time units. These differences allow us to provide an efficient rerouting approach to the wildfire asset protection problem.

\section{Rerouting vehicles during wildfires}

The wind change that occurred during the 2009 Black Saturday fires is one example of the myriad of disruptions that typically occur during wildfire response, requiring replanning and updating of any existing plans. Some of the various disruptions that may occur during wildfires are discussed in more detail next.

The weather - temperature, relative humidity, wind speed and wind direction - affects the direction, intensity and speed at which a wildfire spreads (Sullivan, 2009a,b,c). As such, fire spread predictions are heavily reliant on weather forecasts. A change in wind speed can delay or hasten the fire's anticipated time to impact and hence the required timing of asset protection activities. A change in the direction or extent of fire spread may result in additional assets being impacted, or conversely, assets previously requiring protection may no longer be under threat. A fire burning with a higher intensity than originally predicted may require that additional resources be assigned to the asset in order to provide adequate protection.

Vehicle breakdowns are a common source of disruption in vehicle routing problems. The problem of vehicle breakdowns is exacerbated in the wildfire context by the extreme conditions in which the emergency vehicles must operate. If a vehicle breaks down, it may be necessary to reroute other vehicles to perform 
the tasks of the broken vehicle. In the worst case, due to a lack of resources, it may no longer be possible to adequately protect an asset which previously had sufficient resources assigned to it.

Modified road conditions can result in changes to travel times between locations. For example, roads may become inaccessible due to falling trees, or congested as a result of increased traffic flow as residents evacuate their homes. Increased traffic times may be experienced because of this. Similarly, reduced visibility due to smoke can force responding vehicles to slow down in order to travel safely. Conversely, travel times could be reduced due to road access restrictions whereby roads are closed to the public with access only permitted to emergency vehicles.

Fire trucks and other fire fighting appliances are often reliant on a functioning reticulated water supply. A loss in reticulated water may change the vehicle type required or increase the number of vehicles needed to protect an asset. Additionally, the turn-around time of vehicles between assets may increase, due to the need to source a static water supply prior to moving onto the next protection priority.

The types of disruptions described above by no means represents an exhaustive list. As such, a wildfire rerouting model should also be flexible enough to be able to handle less common and unforeseen disruptions. Furthermore, given the time-critical nature of wildfire response, it is important that asset protection plans are updated and implemented as quickly as possible following a disruption.

There is an incentive for minimising deviations from the original vehicle assignment when rerouting vehicles. Vehicles are dispatched with information about the routes to use, how to protect specific assets and in some cases with specialised equipment. There is also a burden placed on the IMT in terms of communicating and managing the updated plans. Having updated plans as close as possible to the original pre-disruption plans, would result in a reduced burden on the IMT and improved efficiency in the process of updating plans. With this in mind, we investigate three methods for measuring the deviations from the original plans. In preparing a revised asset protection plan, the primary aim is to maximise the value of protected assets. The secondary aim is to minimise deviation from the original plans as represented by the chosen deviation measure. In the next two sections we introduce our approach. In section 3 we present a modified version of the model proposed by Van der Merwe et al. (2015) that we are going to use to maximise protected value. In section 4 we introduce the deviation measures we use to minimise deviation from the pre-disruption vehicle assignment plan.

\section{Vehicle assignment to maximise value protected}

The model formulated here extends the mixed integer programming model presented by Van der Merwe et al. (2015). In their formulation, vehicles are grouped by vehicle type using integer vehicle flow decision variables. Our formulation explicitly keeps track of each vehicle, using binary decision variables to describe each vehicle's individual path. The additional information provided by the binary vehicle flow formulation enables us to model disruptions affecting specific vehicles. Further, we introduce a number of parameters and decision variables to track changes to the routes of vehicles or the vehicle-asset assignments with the goal of measuring deviations from the original pre-disruption vehicle assignments. The following notation is used in the model formulation:

\section{Sets:}

$\mathcal{U} \quad$ is the set of vehicle capabilities.

$\mathcal{P} \quad$ is the set of available vehicles.

$\mathcal{E}_{p} \quad$ is the set of feasible routes for vehicle $p .^{1}$

$\delta_{p}^{+}(k)$ is the set of locations that can be reached directly from location $k$. That is, for each $p$ in $\mathcal{P}, \delta_{p}^{+}(k)=\left\{j \mid(i, j) \in \mathcal{E}_{p}, i=k\right\}$.

$\delta_{p}^{-}(k)$ is the set of locations from where location $k$ can be reached. That is, for each $p$ in $\mathcal{P}, \delta_{p}^{-}(k)=\left\{i \mid(i, j) \in \mathcal{E}_{p}, j=k\right\}$.

\footnotetext{
${ }^{1}$ If $\mathcal{L}$ is the set of all possible location pairs, then for each $p \in \mathcal{P},(i, j) \in \mathcal{E}_{p}$ if and only if $(i, j) \in \mathcal{L}$ and $o_{i}+a_{i}+t_{i j p} \leq c_{j}$.
} 


\section{Parameters:}

$a_{i} \quad$ is the service duration associated with location $i$.

$\boldsymbol{c a p}_{p} \quad$ the capability vector associated with vehicle $p$.

$c_{i} \quad$ is the latest time that protection activities may commence.

$m \quad$ is the number of depots.

$n \quad$ is the number of nodes in the graph representation of the problem.

$o_{i} \quad$ is the earliest time that protection activities may commence.

$\boldsymbol{r}_{i} \quad$ the protection requirement of asset $i$. An asset is protected if the total capabilities provided by assigned vehicles meets or exceeds this protection requirement. For example, the protection requirement $\boldsymbol{r}=(1,4,4)$ can be met by three vehicles with capabilities $\boldsymbol{c a p}_{1}=(0,1,1), \boldsymbol{c} \boldsymbol{a} \boldsymbol{p}_{2}=(1,1,1)$ and $\boldsymbol{c a p}_{3}=(0,2,3)$, respectively.

start $_{i p} 1$ if vehicle $p$ is at depot $i, 0$ otherwise.

$t_{i j p} \quad$ the travel time from location $i$ to location $j$ of vehicle $p$.

$v_{i} \quad$ is the value of the asset at location $i$.

$x_{i j p} \quad 1$ if vehicle $p$ was assigned to travel from location $i$ to location $j$ in the predisruption assignments, 0 otherwise.

\section{Variables:}

$S_{i} \quad$ is the time at which service commences at location $i$.

$X_{i j p} \quad 1$ if vehicle $p$ travels from location $i$ to location $j, 0$ otherwise.

$Y_{i} \quad 1$ if location $i$ is serviced, 0 otherwise.

$Z_{p} \quad 0$ if all assignments of vehicle $p$ remain unchanged in the updated plan, otherwise 1 .

$Z_{p}^{*} \quad 1$ if an asset has been added to or removed from a vehicle's path, 0 otherwise.

$Z_{i p}^{+} \quad 1$ if location $i$ is assigned to vehicle $p$ in the updated plans, but not the predisruption plans, 0 otherwise.

$Z_{i p}^{-} \quad 1$ if location $i$ is assigned to vehicle $p$ in the pre-disruption plans, but not the updated plans, 0 otherwise.

Based on the notation above, the problem of rerouting vehicles to minimise deviation may be formulated as a bi-objective mixed integer programming problem. The primary objective is to maximise the total protected value and the secondary objective is to minimise deviation from the original pre-disruption plans, that is

$$
\text { Maximise } \sum_{i=m+1}^{n-1} v_{i} Y_{i}, \text { Minimise } f_{2}
$$

where $f_{2}$ is the chosen deviation measure.

The assignment of vehicles are subject to the following constraints:

$$
\begin{gathered}
\sum_{j \in \delta_{p}^{+}(i)} X_{i j p}=\operatorname{start}_{i p} \quad \forall i=1, \ldots, m, p \in \mathcal{P} ; \\
\sum_{p \in \mathcal{P}} \sum_{i \in \delta_{p}^{-}(n)} X_{i n p}=|\mathcal{P}| ; \\
\sum_{i \in \delta_{p}^{-}(k)} X_{i k p}=\sum_{j \in \delta_{p}^{+}(k)} X_{k j p} \quad \forall k=m+1, \ldots, n-1, p \in \mathcal{P} ; \\
\sum_{p \in \mathcal{P}} \sum_{i \in \delta_{p}^{-}(k)} X_{i k p} \boldsymbol{c a p}_{p} \geq \boldsymbol{r}_{k} Y_{k} \quad \forall k=m+1, \ldots, n-1 ; \\
S_{i}+t_{i j p}+a_{i}-S_{j} \leq M_{1}\left(1-X_{i j p}\right) \quad \forall(i, j) \in \mathcal{E}_{p}, p \in \mathcal{P} ; \\
o_{i}-S_{i} \leq M_{2}\left(1-Y_{i}\right) \quad \forall i=1, \ldots, n ; \\
S_{i}-c_{i} \leq M_{3}\left(1-Y_{i}\right) \quad \forall i=1, \ldots, n ; \\
Y_{i} \in\{0,1\} \quad \forall i=m+1, \ldots, n-1 ; \\
X_{i j p} \in\{0,1\} \quad \forall p \in \mathcal{P},(i, j) \in \mathcal{E}_{p} ; \\
S_{i} \in \mathbb{R} \quad \forall i=1, \ldots, N .
\end{gathered}
$$

Constraints (2) enforce the starting position of vehicles. Constraint (3) ensures vehicles end at the sink node. The vehicle flow to and from each location is balanced by constraints (4). Constraints (5) enforce the condition that an asset is protected only if the vehicles assigned to the asset collectively meet the protection 
requirement. Constraints (6) ensure that service at a location may only start after protection activity at a previously visited location has been completed and sufficient time for travel has been allowed, with $M_{1}$ representing a large constant. Setting $M_{1}=\max \left(o_{i}\right)+\max \left(t_{i j p}\right)+\max \left(a_{i}\right)-\min \left(c_{i}\right)$ is sufficiently large for this purpose.

The start of protection activities at locations are limited to their respective time windows by constraints (7) and (8). Note that visits to locations are allowed outside of the time windows if they do not contribute to the protected value. This is to allow for original vehicle assignments which may have become infeasible after the disruption due to changes in time windows. These infeasible routes need to be accounted for in order to measure the disruption that occurs when vehicles are rerouted. Setting $M_{2}=M_{3}=\max \left(c_{i}\right)$ is sufficiently large for this purpose.

Constraints (9) and (10), and constraints (11) define the domains of the binary and continuous decision variables, respectively.

Note that the initial locations do not have a service duration associated with them, therefore $a_{i}=0$ for each $i=1, \ldots, m$. Additionally for each initial location $S_{i}=0$ if vehicles are allowed to depart immediately, otherwise, $S_{i}=q_{i}$ where $q_{i}$ is the time that is required before the vehicle at location $i$ is ready for departure.

\section{Measuring deviation}

Recall that the parameter $x_{i j p}$ represents the vehicle assignment before any disruption occurred, $x_{i j p}=1$ if vehicle $p$ travelled from $i$ to $j$ in the initial assignment, otherwise $x_{i j p}=0$. And $\mathcal{P}$ is the set of available vehicles for reassignment.

\subsection{Minimising the number of vehicles with updated routes}

In our first proposed deviation measure, the aim is to minimise the number of vehicles that are affected by planning changes. Let $Z_{p}=0$ if the route associated with vehicle $p$ remains unchanged in the updated plan, otherwise $Z_{p}=1$. Since $X_{i j p}$ represent the new assignment of vehicles, the constraints

$$
X_{i j p}-x_{i j p} \leq Z_{p} \text { and } X_{i j p}-x_{i j p} \geq-Z_{p} \forall(i, j) \in \mathcal{E}_{p}, p \in \mathcal{P} \text {. }
$$

enforce the appropriate values of $Z_{p}$. In this case the deviation minimising objective is given by

$$
\text { Minimise } \sum_{p \in \mathcal{P}} Z_{p}
$$

The complete rerouting model is described by equations (1)-(11),(12), and (13).

4.2 Minimising the number of vehicles with updated routes, allowing changes in servicing sequence

Since a vehicle already has all the information and tools required to provide protection to the assets assigned to it, it may be desirable to allow vehicles to change the sequence in which they provide protection to assets. This will allow greater flexibility in updating vehicle assignments.

Hence in our second deviation measure, the aim is to minimise the number of disrupted vehicles, allowing for changes in route sequence. Let $Z_{p}^{*}=0$ if vehicle $p$ is protecting the same assets, but potentially in a different sequence, 1 otherwise. Note that $\sum_{i=1}^{n-1} X_{i j p}=1$ if vehicle $p$ is assigned to asset $i$ in the updated plans. The parameter $\sum_{i=1}^{n-1} x_{i j p}=1$ if vehicle $p$ was assigned to asset $i$ before any disruptions. Therefore the appropriate value of $Z_{p}^{*}$ is enforced by the constraints

$$
\sum_{i=1}^{n-1} X_{i j p}-\sum_{i=1}^{n-1} x_{i j p} \leq Z_{p}^{*} \text { and } \sum_{i=1}^{n-1} X_{i j p}-\sum_{i=1}^{n-1} x_{i j p} \geq-Z_{p}^{*} \forall j=1, \ldots, n, p \in \mathcal{P} \text {. }
$$

The deviation minimising objective is given by

$$
\text { Minimise } \sum_{p \in \mathcal{P}} Z_{p}^{*}
$$

The complete model is described by equations (1)-(11),(14) and (15). 


\subsection{A general deviation measure}

A limitation of the above proposed deviation measures is that multiple changes to single vehicle paths are counted as single changes. In some case it may be desirable to make a couple of minor changes to multiple vehicles to deal with a disruption instead of making major changes to the assignment of a single vehicle. In such a case the main concern would be to maintain vehicle-asset pair assignment while allowing partial changes to a vehicle path.

Let $Z_{i p}^{+}=1$ if location $i$ is assigned to vehicle $p$ in the updated plans, but not the pre-disruption plans, 0 otherwise. Let $Z_{i p}^{-}=1$ if location $i$ is assigned to vehicle $p$ in the pre-disruption plans, but not the updated plans, 0 otherwise. These values are enforced by the constraints

$$
\sum_{i=1}^{n-1} X_{i j p}-\sum_{i=1}^{n-1} x_{i j p}=Z_{i p}^{+}-Z_{i p}^{-} \forall j \in m+1, \ldots, n, p \in \mathcal{P}
$$

The cost of reassigning a vehicles may be a dependent on a variety of factors related to the specific asset and vehicle in question. The following parameters allow an IMT flexibility when considering various assignment changes, allowing the IMT to weigh each change in the objective function to reflect these costs or the IMT's own management priorities. Let $\operatorname{cost}_{i p}^{+}$be the cost of adding asset $i$ to vehicle $p$ 's assignment, and let $\operatorname{cost}_{i p}^{-}$be the cost of removing asset $i$ from vehicle $p$ 's assignment. The deviation minimising objective is given by

$$
\text { Minimise } \sum_{p \in \mathcal{P}} \sum_{i=1}^{N} \operatorname{cost}_{i p}^{+} Z_{i p}^{+}+\operatorname{cost}_{i p}^{-} Z_{i p}^{-} \text {. }
$$

The complete model is described by equations (1)-(11), (16) and (17). Note that if $\operatorname{cost}_{i p}^{+}=\operatorname{cost}_{i p}^{-}=1$, then the number of asset-vehicle reassignments are minimised. Setting $\operatorname{cost}_{i p}^{+}=0$ (or $\left.\operatorname{cost}_{i p}^{+}\right)$would mean that adding (or removing) and asset-vehicle assignment pair from the assignment plans won't contribute to the deviation measure.

\section{Model implementation and demonstration}

We use the algorithm proposed by Özlen and Azizoğlu (2009) to determine all non-dominated solutions to our bi-objective integer programming problem. The algorithm is based on the classical $\epsilon$-constraint method (Haimes et al., 1971). The rerouting problem is implemented as a single objective MIP, maximising the objective $f_{1}-\epsilon f_{2}$, where $f_{1}$ is the primary objective to be maximised, $f_{2}$ the secondary objective to be minimised, and $\epsilon$ is a scaling factor which enforces the lexicographical ordering on the objective functions. A constraint specifying an upper bound on the secondary objective is iteratively decreased until all nondominated solutions have been found. In the case of the first deviation measure, the constraint $\sum_{p \in \mathcal{P}} Z_{p} \leq \bar{P}$ is added, where $\bar{P}$ is the upper bound that is decreased in each iteration.

Next, we demonstrate the model with the aid of a wildfire scenario in which an unforeseen change in weather occurs. In the scenario, a wildfire ignites south of Hobart, Tasmania, Australia. A fire spread forecast is generated taking into account the current, expected weather conditions. The location of the fire, together with the initial fire spread prediction, is shown in Figure 1. Realistic fire spread data, provided by the Tasmania Fire Service, is used in this demonstration.

\subsection{Initial assignment}

The fire, illustrated in Figure 2, is expected to impact 22 community assets listed in Table 1 . Fire crews operating various fire fighting vehicle types, i.e. medium/heavy pumpers and light/medium/heavy tankers are available to respond to the wildfire to provide protection to the community assets. There are 25 vehicles in total, each vehicle is stationed at one of 10 fire stations, listed in Table 2. The protection requirement descriptions are based on expert opinion (C. Collins, Tasmania Fire Service, personal communication, 2015). The capability vectors of vehicles and protection requirement vectors of locations were derived from these protection requirement descriptions, shown in Table 1 . The derived vehicle capability vectors are $\operatorname{cap}_{H P}=$ $(1,0,0,0,0), \operatorname{cap}_{M P}=(1,0,0,0,0), \operatorname{cap}_{H T}=(0,1,1,0,0), \operatorname{cap}_{M T}=(0,0,1,0,1)$ and $\operatorname{cap}_{L T}=(0,0,0,1,1)$. Driving times were taken from Google's Distance Matrix Service API.

It is assumed that the service duration of each asset is 30 minutes. The value of assets were picked, with equal probability, from the set $\{10,20,30\}$, in reality these values are determined by planning bodies before 


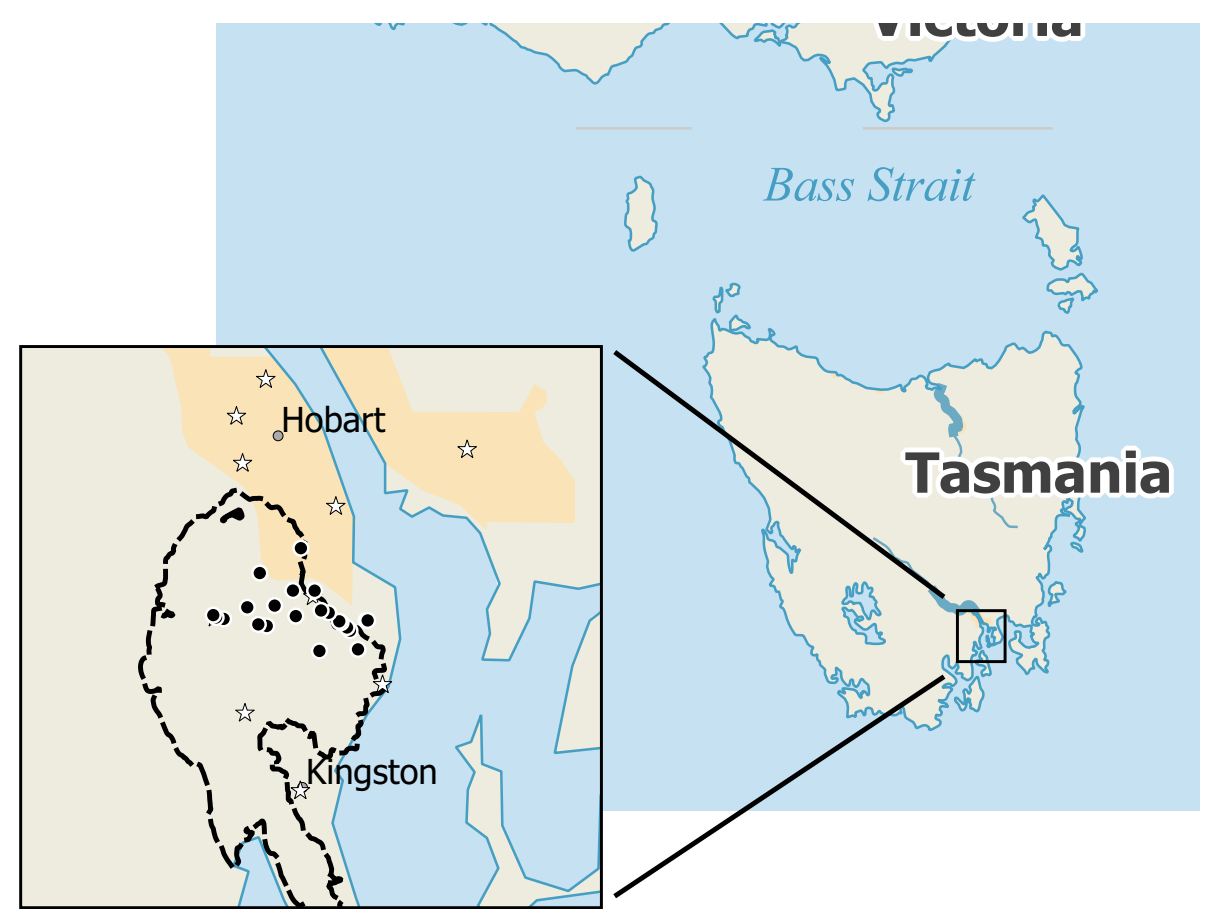

Fig. 1 The study area is located near Hobart, Tasmania, Australia. The perimeter of the initial expected spread fire is indicated by a dashed contour, the locations of fire stations are indicated by $\star$, and community assets are indicated by $\bullet$.

any wildfire commences, or IMTs during the management of a wildfire. Optimising the resource allocation results in the assignments shown in Table 3. All the assets can be protected with the 25 available vehicles in the initial assignment.

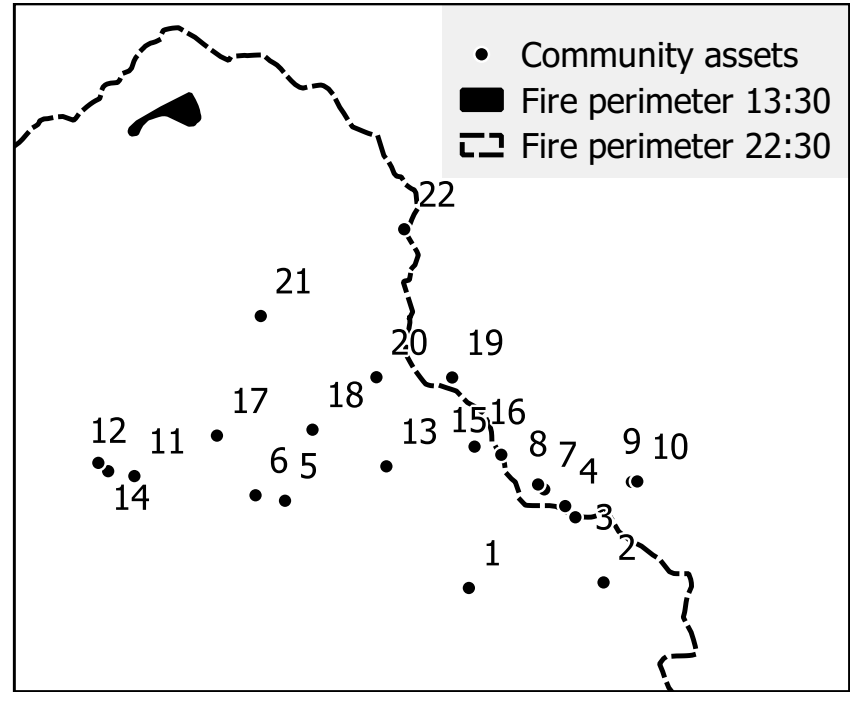

Fig. 2 The initial fire spread prediction. A number of community assets located in South Hobart are labelled 1 through 22. The time to impact and protection requirements for each asset are listed in Table 1.

\begin{tabular}{rrrr}
\hline Vehicle & $\begin{array}{l}\text { Assigned } \\
\text { assets }\end{array}$ & Vehicle & $\begin{array}{c}\text { Assigned } \\
\text { assets }\end{array}$ \\
\hline 1 & - & 14 & 4,22 \\
2 & - & 15 & 12 \\
3 & - & 16 & - \\
4 & 15,18 & 17 & - \\
5 & 17,22 & 18 & 1,18 \\
6 & 17,20 & 19 & - \\
7 & 16,18 & 20 & 21 \\
8 & 14,21 & 21 & - \\
9 & 2 & 22 & 18 \\
10 & 5,19 & 23 & 19 \\
11 & 1,3 & 24 & 4,22 \\
12 & 11,13 & 25 & 17 \\
13 & 6 & & \\
\hline
\end{tabular}

Table 3 The initial vehicle assignment.

\subsection{Disruption}

A change in the forecasted weather is observed after planning for the initial expected weather conditions is completed and vehicles have been dispatched. The changed, disrupted, fire spread perimeter is shown in 


\begin{tabular}{|c|c|c|c|c|}
\hline Asset & Described & Vector & Original & Changed \\
\hline 2 Transend Communications Site & $1 \times \mathrm{HT} / \mathrm{MT}$ & $(0,0,1,0,0)$ & 215 & 179 \\
\hline 3 Communications Tower & $1 \times \mathrm{HT} / \mathrm{MT}$ & $(0,0,1,0,0)$ & 219 & 182 \\
\hline 4 Broughton Ave Reservoir & $1 \times \mathrm{LT} / \mathrm{MT}$ & $(0,0,0,0,1)$ & 203 & 189 \\
\hline 7 Communications Exchange & $1 \times \mathrm{LT} / \mathrm{MT}$ & $(0,0,0,1,0)$ & $\mathrm{N} / \mathrm{A}$ & 185 \\
\hline 8 Arts Centre & $1 \times \mathrm{HP} / \mathrm{MP}$ & $(1,0,0,0,0)$ & $\mathrm{N} / \mathrm{A}$ & 185 \\
\hline 9 The Signalmans Cottage & $\mathrm{HP} / \mathrm{MP} \& \mathrm{MT} / \mathrm{LT}$ & $(1,0,1,0,0)$ & $\mathrm{N} / \mathrm{A}$ & 144 \\
\hline 10 Communications Towers & $1 \times \mathrm{HT} / \mathrm{MT}$ & $(0,0,1,0,0)$ & $\mathrm{N} / \mathrm{A}$ & 144 \\
\hline 11 Mountain Lodge & $1 \times \mathrm{HT} / \mathrm{MT}$ & $(0,0,1,0,0)$ & 177 & $\mathrm{~N} / \mathrm{A}$ \\
\hline 12 Telstra Fern Tree Exchange & $1 \times \mathrm{LT} / \mathrm{MT}$ & $(0,0,0,0,1)$ & 180 & $\mathrm{~N} / \mathrm{A}$ \\
\hline 17 Communications Tower (Chimney Pot Hill) & $1 \times \mathrm{HT} \& 1 \times \mathrm{LT} / \mathrm{MT}$ & $(0,1,0,0,1)$ & 87 & 167 \\
\hline 18 Pump Station (Potable Water) & $2 \times \mathrm{LT} / \mathrm{MT}$ & $(0,0,0,0,2)$ & 110 & 119 \\
\hline 19 Communications Tower (Olinda Grove) & $1 \times \mathrm{HT}$ & $(0,1,0,0,0)$ & $\mathrm{N} / \mathrm{A}$ & 145 \\
\hline 20 Communications Tower (Tolmans Hill) & $1 \times \mathrm{HT}$ & $(0,1,0,0,0)$ & 160 & 133 \\
\hline 21 HCC Mountain Park Depot & $1 \times \mathrm{HT} \& 1 \times \mathrm{LT} / \mathrm{MT}$ & $(0,1,0,0,1)$ & 90 & 93 \\
\hline 22 Cascade Hotel & $1 \times \mathrm{HP} / \mathrm{MP}$ & $(1,0,0,0,0)$ & 141 & 128 \\
\hline
\end{tabular}

Table 1 Community assets, their protection requirements, and the time to impact in minutes. The time to impact is measured from the time the fire is ignited; 'N/A' indicates that the asset is not being impacted and does not require any protection. Two protection requirements are shown, the 'described' column shows the description in terms of number and type of trucks, the 'vector' column shows the parametrised vector representation of the protection requirement. The abbreviations have the following meanings: HP - heavy pumper, MP - medium pumper, HT - heavy tanker, MT - medium tanker, and LT - light tanker.

\begin{tabular}{lccccc}
\hline Brigade & HP & MP & HT & MT & LT \\
\hline 1 Fern Tree & - & - & - & 2 & 1 \\
2 Hobart-Glenorchy & 1 & - & - & - & 2 \\
3 Hobart & 2 & - & - & - & 3 \\
4 Hobart-Clarence & 1 & - & - & 1 & 1 \\
5 Hobart-Taroona & - & - & - & 1 & 1 \\
6 Hobart-Lenah Valley & - & - & - & - & - \\
7 Hobart-MT Nelson & - & - & 1 & - & 1 \\
8 Kingston & - & 1 & 1 & 1 & 1 \\
9 Summerleas & - & - & - & 1 & - \\
10 Wellington & - & - & 1 & - & 1 \\
\hline Total: $(=\mathbf{2 5})$ & 4 & 1 & 3 & 6 & 11 \\
\hline
\end{tabular}

Table 2 Fire stations located near South Hobart and the number of vehicles of each type stationed at the fire stations.

Figure 3. The new fire spread prediction shares ignition conditions with the initial expected fire spread. The disrupted time to impact is shown in the last column of Table 1. The change in the weather conditions results in changes to the expected time to impact for most locations. A number of the assets are no longer being impacted, whereas locations not threatened previously are being impacted in the new conditions. It is assumed that the protection requirement of each asset is unchanged.

\subsection{Rerouting vehicles}

The Pareto-optimal results when rerouting vehicles and minimising the number of changes to vehicle paths, i.e. considering secondary objective (13), are plotted in Figure 4. The plot shows that increasing the number of changes made to the vehicle assignments results in an increase of the total value of assets protected. If no vehicles are rerouted after the disruption occurs, then the total protected asset value is $65 \%$ of the highest possible value that can be protected. However, not all the vehicle routes have to be changed to reach this maximum protection level, rerouting nine vehicles is sufficient (shown in Table 4).

It took on average 5 minutes to generate a complete Pareto-frontier for each deviation measure. The problem was solved on a desktop computer with an Intel i7 processor and 8GB of RAM. The models were 


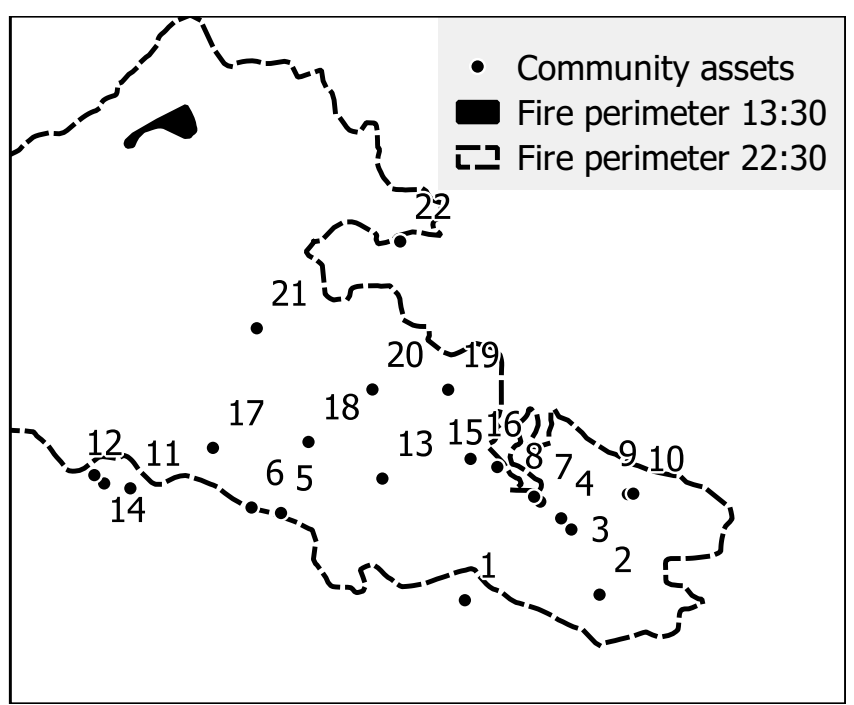

Fig. 3 The updated fire spread prediction. A number of community located in South Hobart are labelled 1 through 22. The time to impact and protection requirements for each location are listed in Table 1 .

\begin{tabular}{rrrr}
\hline Vehicle & $\begin{array}{l}\text { Assigned } \\
\text { assets }\end{array}$ & Vehicle & $\begin{array}{c}\text { Assigned } \\
\text { assets }\end{array}$ \\
\hline 1 & - & $\mathbf{1 4}$ & $\mathbf{1 0 , 2 1}$ \\
2 & - & 15 & 12 \\
$\mathbf{3}$ & $\mathbf{8 , 9 , 2 1}$ & 16 & - \\
$\mathbf{4}$ & $\mathbf{1 5 , 2 1}$ & 17 & - \\
$\mathbf{5}$ & $\mathbf{2 2}$ & $\mathbf{1 8}$ & $\mathbf{7 , 1 8}$ \\
$\mathbf{6}$ & $\mathbf{3 , 2 0}$ & 19 & - \\
$\mathbf{7}$ & $\mathbf{1 6}$ & 20 & 21 \\
$\mathbf{8}$ & $\mathbf{5 , 1 7 , 2 1}$ & 21 & - \\
9 & 2 & 22 & 18 \\
10 & 5,19 & 23 & 19 \\
$\mathbf{1 1}$ & $\mathbf{9}$ & 24 & 4,22 \\
12 & 11,13 & 25 & 17 \\
13 & 6 & & \\
\hline
\end{tabular}

Table 4 The changed vehicle assignment to account for the disruption. Vehicles that have been reassigned are indicated by boldface entries.

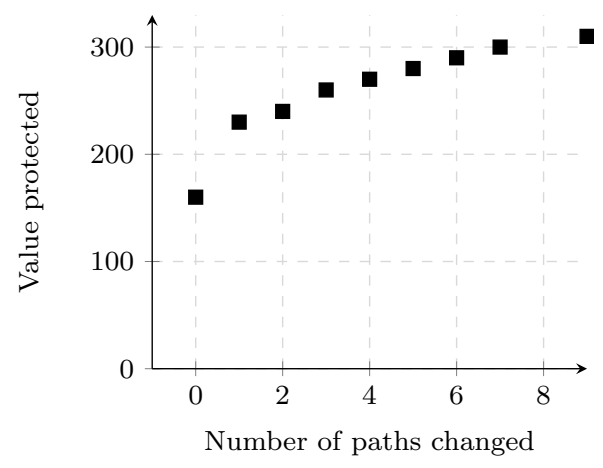

Fig. 4 The Pareto-optimal solutions for the weather change scenario when minimising the changes to vehicle paths.

described in CMPL (Schleiff and Steglich, 2015) and solved using CPLEX 12.6 (IBM Corporation, 2015). We investigate the computational behaviour of the model in the next section.

\section{Computational testing}

Computational testing was carried out on a single node of a computer cluster. The node has two Intel Xeon E5-2670 processors and 64GB of RAM. The problem instances were solved using CPLEX 12.6 and performance was measured in elapsed time (wall clock time), a time limit of 30 minutes (1800 seconds) was set.

The problem instances proposed by Van der Merwe et al. (2015) were considered. These problem instances range in size from 30 to 60 assets with 10 vehicles available for assignment. The length $w$ of time windows in an instance are either all equal to 20 or 40 minutes.

An initial, pre-disruption vehicle assignment is created by solving these problem instances in the absence of any disruption. Vehicles are then rerouted to account for the breakdown of a single vehicle. The breakdown vehicle is chosen at random. The third deviation measure is considered, minimising the number of vehicle-asset assignment changes and the time required to find a single optimal solution, allowing maximum deviation, is reported.

The average time required to find an optimal solution for each set of instances is shown in Table 5 . Problems with 30 assets and small time windows yield optimal solutions in a matter of seconds. We expect that generally practical problems with 30 or less asset and small time windows would be quick to solve to optimality using the current approach. Solving problems of size 40 with small time windows may still be feasible in practice, but result in an average solution time of 10 minutes. However, for larger time windows 
and more than 40 assets, alternative solution methods or heuristics will be required to provide solutions within a reasonable time frame.

\begin{tabular}{rrr}
\hline & \multicolumn{2}{c}{ Solution time } \\
Size & $w=20$ & $w=40$ \\
\hline 30 & $13(0 \%)$ & $1166(2.5 \%)$ \\
40 & $660(0.7 \%)$ & $-(11.4 \%)$ \\
50 & $1488(3.2 \%)$ & $-(22.0 \%)$ \\
60 & $-(10.2 \%)$ & $-(37.6 \%)$ \\
\hline
\end{tabular}

Table 5 The solution times for test instances in seconds. The average relative optimality gap is indicated in brackets. A dash (-) indicates that none of the problems could be solved to optimality within the 30 minute time limit. Feasible solutions were found for all test instances considered.

\section{Discussion}

In this paper we presented a method for rerouting vehicles to adapt to changes during wildfire asset protection. A number of potential secondary objectives were considered to minimise changes made to the assignment of vehicles. These deviation measures provide a varying degree of flexibility when rerouting vehicles, the specific deviation measure chosen would depend on the priority and objectives of the IMT.

The rerouting framework presented here allows for the consideration of a variety of disruptions that can occur when assigning vehicles to asset protection activities. An unexpected weather disruption case study was presented using a wildfire scenario in South Hobart. Vehicle breakdowns can be considered by removing the vehicle from the set $\mathcal{P}$ and then reassigning the subset of available vehicles. If a vehicle cannot be rerouted, then its route can be fixed by adding constraints of the type $X_{i j p}=x_{i j p}$ for the appropriate edges and vehicle.

Although the discussion focused on asset protection, other tasks performed by wildfire resources can also be accommodated by the modelling approach presented here. For example, assisting with the evacuation of people, collecting information or aiding with direct suppression activities can be parametrised without any changes to the model by associating a value with the successful completion of the activity. A mandatory task at location $i$ can be considered by adding the constraint $Y_{i}=1$.

Computational testing showed that realistic sized problems can be solved within a reasonable time. Although larger problems weren't solved to optimality, feasible solutions were found for these problems. To address the quality of solutions, future work into efficient solution methods to solve larger problems in a short time frame would be valuable. Murray and Karwan (2013) used a branch and bound method to solve a similar rerouting model. Modifications to this approach could make it applicable to the problem presented here.

In some cases there may be some expectation of the disruption. For example, if the likelihood of weather outcomes are known, then combining a rerouting approach with a stochastic programming approach could lead to improved vehicle assignments. The problem would then be formulated as a two-stage stochastic programming model, with initial vehicle assignments made in the first stage with the opportunity for adjustments in the second stage based on observed fire-weather outcomes.

Changes and disruptions during wildfires are often difficult to forecast. For example, predicting when and where a truck will break down, or forecasting the exact nature of changes to the weather are inherently difficult. Updating plans are thus often reactionary by nature and rerouting vehicles may be the only option available. Our approach quickly provides a set of solutions, allowing the IMT to choose the most appropriate assignment plan.

\section{Acknowledgements}

We would like to thank Damien Killalea and Chris Collins from the Tasmania Fire Service for valuable discussions and information provided on the problems faced in wildfire incident management. This work was supported by the Bushfire CRC in the form of scholarship funding to Martijn van der Merwe. Melih Ozlen is supported by the Australian Research Council under the Discovery Projects funding scheme (project DP140104246). Computational testing was carried out on Trifid, a high performance computer cluster belonging to V3 Alliance. 


\section{References}

Australasian Fire Authorities Council (2013). The Australasian Inter-service Incident Management System. 4th edition. AFAC: East Melbourne.

Cruz M, Sullivan A, Gould J, Sims N, Bannister A, Hollis J and Hurley R (2012). Anatomy of a catastrophic wildfire: The Black Saturday Kilmore East fire in Victoria, Australia. Forest Ecology and Management 284: 269-285. doi:10.1016/j.foreco.2012.02.035.

Haimes, Y Y, Lasdon, L S and Wismer, D A (1971). On a Bicriterion Formulation of the Problems of Integrated System Identification and System Optimization. IEEE Transactions on Systems Man and Cybernetics 1(3): 296-297 doi:10.1109/TSMC.1971.4308298.

IBM Corporation (2015). CPLEX Software, Version 12.6. Armonk, New York.

ICS Canada (2012). Incident command system operational description. http://www.icscanada.ca/ accessed 19 September 2013.

Mamasis K, Minis I and Dikas G (2012). Managing vehicle breakdown incidents during urban distribution of a common product. Journal of the Operational Research Society 64(6): 925-937. doi:10.1057/jors.2012.93.

Minis I, Mamasis K and Zeimpekis V (2011). Real-time management of vehicle breakdowns in urban freight distribution. Journal of Heuristics 18(3): 375-400. doi:10.1007/s10732-011-9191-1.

Murray C and Karwan M (2013). A branch-and-bound-based solution approach for dynamic rerouting of airborne platforms. Naval Research Logistics (NRL) 60(2): 141-159. doi:10.1002/nav.21526.

Murray C C and Karwan M H (2010). An extensible modeling framework for dynamic reassignment and rerouting in cooperative airborne operations. Naval Research Logistics (NRL) 57(7): 634-652. doi:10.1002/ nav.20427.

Özlen M and Azizoğlu M (2009). Multi-objective integer programming: A general approach for generating all non-dominated solutions. European Journal of Operational Research 199(1): 25-35 doi:10.1016/j.ejor. 2008.10.023.

Pillac V, Gendreau M, Guéret C and Medaglia A L (2013). A review of dynamic vehicle routing problems. European Journal of Operational Research 225(1): 1-11. doi:10.1016/j.ejor.2012.08.015.

Schleiff T and Steglich M (2015). CMPL Software, Version 1.10. https://projects.coin-or.org/Cmpl accessed 11 May 2015.

Sullivan A L (2009a). Wildland surface fire spread modelling, 1990-2007. 1: Physical and quasi-physical models. International Journal of Wildland Fire 18(4): 349-368. doi:10.1071/WF06143.

Sullivan A L (2009b). Wildland surface fire spread modelling, 1990-2007. 2: Empirical and quasi-empirical models. International Journal of Wildland Fire 18(4): 369-386. doi:10.1071/WF06142.

Sullivan A L (2009c). Wildland surface fire spread modelling, 1990-2007. 3: Simulation and mathematical analogue models. International Journal of Wildland Fire 18(4): 387-403. doi:10.1071/WF06144.

US Department of Homeland Security (2008). National Incident Management System. US Government: Washington, DC.

Van der Merwe M, Minas J P, Ozlen M and Hearne J W (2015). A mixed integer programming approach for asset protection during escaped wildfires. Canadian Journal of Forest Research 45(4): 444-451. doi: 10.1139/cjfr-2014-0239. 